\title{
A note on complete convergence of weighted sums for array of rowwise AANA random variables
}

\author{
Xinghui Wang, Aiting Shen and Xiaoqin Li
}

\section{"Correspondence:}

lixiaoqin1983@163.com

School of Mathematical Science,

Anhui University, Hefei, 230039, P.R.

China

\begin{abstract}
In this paper, we consider complete convergence and complete moment convergence of weighted sums for an array of rowwise AANA random variables. The main result of the paper generalizes the Baum-Katz theorem on AANA random variables. Our results extend and improve the corresponding ones of Wang et al. (Abstr. Appl. Anal. 2012:315138, 2012).
\end{abstract}

MSC: 60B10; 60F15

Keywords: complete convergence; Baum-Katz theorem; AANA random variable; Marcinkiewicz-Zygmund type strong law of large numbers

\section{Introduction}

Assume that random variables $X_{n}, n \in \mathbb{N}=\{1,2, \ldots\}$ are defined on a fixed probability space $(\Omega, \mathcal{A}, P)$.

First, we recall two definitions as follows.

Definition 1.1 Random variables $X_{1}, X_{2}, \ldots, X_{n}, n \geq 2$, are said to be negatively associated (NA, in short) if

$$
\operatorname{Cov}\left(f\left(X_{i_{1}}, \ldots, X_{i_{k}}\right), g\left(X_{j_{1}}, \ldots, X_{j_{m}}\right)\right) \leq 0
$$

for any pair of nonempty disjoint subsets $A=\left\{i_{1}, \ldots, i_{k}\right\}$ and $B=\left\{j_{1}, \ldots, j_{m}\right\}, k+m \leq n$, of the set $\{1,2, \ldots, n\}$ and for any bounded coordinatewise increasing real functions $f\left(x_{i_{1}}, \ldots, x_{i_{k}}\right)$ and $g\left(x_{j_{1}}, \ldots, x_{j_{m}}\right), x_{1}, \ldots, x_{n} \in \mathbb{R}=(-\infty, \infty)$. Random variables $X_{n}, n \in \mathbb{N}$, are NA if every $n \in \mathbb{N}$ random variables $X_{1}, X_{2}, \ldots, X_{n}$ are NA.

Random variables $X_{n i}, i, n \in \mathbb{N}$, are called an array of rowwise NA random variables if for every $n \in \mathbb{N}$ random variables $X_{n i}, i \in \mathbb{N}$ are, NA.

The concept of NA random variables was introduced by Block et al. [1] and carefully studied by Joav-Dev and Proschan [2]. Primarily motivated by this, Chandra and Ghosal $[3,4]$ introduced the following dependence.

Definition 1.2 Random variables $X_{n}, n \in \mathbb{N}$, are said to be asymptotically almost negatively associated (AANA, in short) if there exists a nonnegative sequence $q(n) \rightarrow 0$ as

๑ 2013 Wang et al.; licensee Springer. This is an Open Access article distributed under the terms of the Creative Commons Attribution License (http://creativecommons.org/licenses/by/2.0), which permits unrestricted use, distribution, and reproduction in any medium, provided the original work is properly cited. 
$n \rightarrow \infty$ such that

$$
\operatorname{Cov}\left(f\left(X_{n}\right), g\left(X_{n+1}, \ldots, X_{n+k}\right)\right) \leq q(n)\left\{\operatorname{Var}\left(f\left(X_{n}\right)\right) \operatorname{Var}\left(g\left(X_{n+1}, \ldots, X_{n+k}\right)\right)\right\}^{1 / 2}
$$

for all $n, k \in \mathbb{N}$ and for all coordinatewise nondecreasing continuous functions $f$ and $g$ for which $\operatorname{Var} f\left(X_{n}\right)$ and $\operatorname{Var} g\left(X_{n+1}, \ldots, X_{n+k}\right)$ exist.

Random variables $X_{n i}, i, n \in \mathbb{N}$, are called an array of rowwise AANA random variables if for every $n \in \mathbb{N}$, random variables $X_{n i}, i \in \mathbb{N}$, are AANA.

The family of AANA random variables contains NA (in particular, independent) random variables (with $q(n)=0, n \geq 1$ ) and some more kinds of random variables which are not much deviated from being negatively associated. An example of AANA random variables which are not NA was constructed by Chandra and Ghosal [3]. For various results and applications of AANA random variables, one can refer to Chandra and Ghosal [4], Wang et al. [5], Ko et al. [6], Yuan and An [7], Wang et al. [8, 9] and Wang et al. [10], Yang et al. [11], Shen and Wu [12] among others.

The concept of complete convergence was introduced by Hsu and Robbins [13] as follows. Random variables $U_{n}, n \in \mathbb{N}$, are said to converge completely to a constant $C$ if $\sum_{n=1}^{\infty} P\left(\left|U_{n}-C\right|>\varepsilon\right)<\infty$ for all $\varepsilon>0$. In view of the Borel-Cantelli lemma, this implies that $U_{n} \rightarrow C$ almost surely (a.s.). The converse is true if random variables $U_{n}, n \in \mathbb{N}$, are independent. Hsu and Robbins [13] proved that arithmetic means of independent and identically distributed (i.i.d.) random variables converges completely to the expected value if the variance of the summands is finite. Erdös [14] proved the converse. The result of Hsu, Robbins and Erdös is a fundamental theorem in probability theory and has been generalized and extended in several directions by many authors. One of the most important generalizations was provided by Baum and Katz [15] for the strong law of large numbers as follows.

Theorem A Let $1 / 2<\alpha \leq 1$ and $\alpha p>1$. Let $X_{n}, n \in \mathbb{N}$ be i.i.d. random variables with zero means. Then the following statements are equivalent:

(i) $E\left|X_{1}\right|^{p}<\infty$,

(ii) $\sum_{n=1}^{\infty} n^{\alpha p-2} P\left(\max _{1 \leq j \leq n}\left|\sum_{i=1}^{j} X_{i}\right|>\varepsilon n^{\alpha}\right)<\infty$ for all $\varepsilon>0$.

Motivated by Baum and Katz [15] for i.i.d. random variables, many authors studied the Baum-Katz-type theorem for dependent random variables. One can refer to Peligrad [16], Shao [17], Peligrad and Gut [18], Kruglov et al. [19], Wang and Hu [20], Shen et al. [21], Wang et al. [22], etc.

Next, we will give the definition of stochastic domination which is used frequently in the paper.

Definition 1.3 Random variables $X_{n}, n \in \mathbb{N}$, are said to be stochastically dominated by a random variable $X$ if for every $n \in \mathbb{N}$ there exists a positive constant $C$ such that

$$
P\left(\left|X_{n}\right|>x\right) \leq C P(|X|>x)
$$

for all $x \geq 0$. 
An array of rowwise random variables $X_{n i}, i, n \in \mathbb{N}$, is said to be stochastically dominated by a random variable $X$ if for every $n \in \mathbb{N}$ there exists a positive constant $C$ such that

$$
\sup _{i \geq 1} P\left(\left|X_{n i}\right|>x\right) \leq C P(|X|>x)
$$

for all $x \geq 0$.

Wang et al. [10] discussed the complete convergence for an array of rowwise AANA random variables which are stochastically dominated by a random variable $X$ and obtained the following result.

Theorem B Let $X_{n i}, i, n \in \mathbb{N}$, be an array of rowwise AANA random variables which are stochastically dominated by a random variable $X$ and $E X_{n i}=0$ for every $i, n \in \mathbb{N}$ with $q(n)$ from Definition 1.2.

(i) Let $1 / 2<\alpha \leq 1, p>1$ and $\alpha p>1$. If $E|X|^{p}<\infty$ and $\sum_{n=1}^{\infty} q^{s / r}(n)<\infty$ for some $r \in\left(3 \cdot 2^{k-1}, 4 \cdot 2^{k-1}\right]$ and

$$
r>\max \left(2, \frac{\alpha p-1}{\alpha-1 / 2}, p\right)
$$

where integer number $k \geq 1$ and $s \doteq r /(r-1)$ for $r>1$, then for all $\varepsilon>0$,

$$
\sum_{n=1}^{\infty} n^{\alpha p-2} P\left(\max _{1 \leq j \leq n}\left|\sum_{i=1}^{j} X_{n i}\right|>\varepsilon n^{\alpha}\right)<\infty
$$

(ii) If $E|X| \log |X|<\infty$ and $\sum_{n=1}^{\infty} q^{2}(n)<\infty$, then for all $\varepsilon>0$,

$$
\sum_{n=1}^{\infty} n^{-1} P\left(\max _{1 \leq j \leq n}\left|\sum_{i=1}^{j} X_{n i}\right|>\varepsilon n\right)<\infty .
$$

The complete convergence for an array of rowwise random variables was studied by many authors. See, for example, the complete convergence for an array of rowwise independent random variables was studied by Hu et al. [23], Sung et al. [24], Kruglov et al. [19] and others. Recently, many authors extended the complete convergence for an array of rowwise independent random variables to the cases of dependent random variables. One can refer to Kuczmaszewska [25, 26], Chen et al. [27], Kruglov [28], Zhou and Lin [29], Guo [30], Wu [31], and so on.

The main purpose of the paper is to further study the complete convergence and complete moment convergence of weighted sums for an array of rowwise AANA random variables. The result of the paper generalizes the Baum-Katz theorem on AANA random variables in different methods. As an application, we get the Marcinkiewicz-Zygmund type strong law of large numbers for weighted sums on AANA random variables. Our results extend and improve the corresponding ones of [10].

Throughout this paper, for $r>1$, let $s \doteq r /(r-1)$ be the dual number of $r$. The symbols $C, C_{1}, C_{2}, \ldots$ denote positive constants which may be different in various places. Assume that $I(A)$ is the indicator function of the set $A$. Let $x^{+}=\max (0, x)$ and $\log x=\ln \max (x, e)$, where $\ln x$ denotes the natural logarithm. $a_{n}=O\left(b_{n}\right)$ stands for $\left|a_{n}\right| \leq C\left|b_{n}\right|$. 


\section{Preliminaries}

To prove the main results of the paper, we need the following lemmas.

Lemma 2.1 (cf. [32, Lemma 4.1.6]) Let $X_{n}, n \in \mathbb{N}$, be random variables, which are stochastically dominated by a random variable $X$. Then, for any $a>0$ and $b>0$, the following two statements hold:

$$
E\left|X_{n}\right|^{a} I\left(\left|X_{n}\right| \leq b\right) \leq C_{1}\left\{E|X|^{a} I(|X| \leq b)+b^{a} P(|X|>b)\right\}
$$

and

$$
E\left|X_{n}\right|^{a} I\left(\left|X_{n}\right|>b\right) \leq C_{2} E|X|^{a} I(|X|>b),
$$

where $C_{1}$ and $C_{2}$ are positive constants.

Lemma 2.2 ( $c f$. [7, Lemma 2.1]) Let $X_{n}, n \in \mathbb{N}$, be AANA random variables with $q(n)$ from Definition 1.2. Assume that $f_{n}, n \in \mathbb{N}$ are all nondecreasing (or all nonincreasing) and continuous functions, then $f_{n}\left(X_{n}\right), n \in \mathbb{N}$, are still AANA random variables with $q(n)$.

Lemma 2.3 ( $c f$. [7, Theorem 2.1]) Let $r>1$ and $X_{n}, n \in \mathbb{N}$, be AANA random variables with $q(n)$ from Definition 1.2.

If $\sum_{n=1}^{\infty} q^{2}(n)<\infty$, then there exists a positive constant $C_{r}$ depending only on $r$ such that for all $n \geq 1$ and $1<r \leq 2$,

$$
E\left(\max _{1 \leq j \leq n}\left|\sum_{i=1}^{j} X_{i}\right|^{r}\right) \leq C_{r} \sum_{i=1}^{n} E\left|X_{i}\right|^{r} .
$$

If $\sum_{n=1}^{\infty} q^{s / r}(n)<\infty$ for some $r \in\left(3 \cdot 2^{k-1}, 4 \cdot 2^{k-1}\right]$, where integer number $k \geq 1$, then there exists a positive constant $D_{r}$ depending only on $r$ such that for all $n \geq 1$,

$$
E\left(\max _{1 \leq j \leq n}\left|\sum_{i=1}^{j} X_{i}\right|^{r}\right) \leq D_{r}\left\{\sum_{i=1}^{n} E\left|X_{i}\right|^{r}+\left(\sum_{i=1}^{n} E X_{i}^{2}\right)^{r / 2}\right\}
$$

Lemma 2.4 (cf. [33, Lemma 2.4]) Let $Y_{n}, Z_{n}, n \in \mathbb{N}$ be random variables. Then, for any $q>1, \varepsilon>0$ and $a>0$,

$$
E\left(\max _{1 \leq j \leq n}\left|\sum_{i=1}^{j}\left(Y_{i}+Z_{i}\right)\right|-\varepsilon a\right)^{+} \leq\left(\frac{1}{\varepsilon^{q}}+\frac{1}{q-1}\right) \frac{1}{a^{q-1}} E \max _{1 \leq j \leq n}\left|\sum_{i=1}^{j} Y_{i}\right|^{q}+E \max _{1 \leq j \leq n}\left|\sum_{i=1}^{j} Z_{i}\right| .
$$

\section{Main results and their proofs}

In this section, let $X_{n i}, i, n \in \mathbb{N}$, be an array of rowwise AANA random variables, i.e., for every $n \in \mathbb{N}, X_{n i}, i \in \mathbb{N}$, are AANA random variables with the identical mixing coefficient $q(i)$ and let $a_{n i}, i, n \in \mathbb{N}$, be an array of real numbers. Let $X_{n}, n \in \mathbb{N}$, be AANA random variables with $q(n)$ from Definition 1.2.

In the following, let $\psi(x)=1$ or $\psi(x)=\log x$. Note that the function $\psi(x)$ has the following properties (see [34]): 
(a) for all $m \geq k \geq 1$,

$$
\sum_{n=k}^{m} n^{r-1} \psi(n) \leq C m^{r} \psi(m) \quad \text { if } r>0
$$

and

$$
\sum_{n=m}^{\infty} n^{r-1} \psi(n) \leq C m^{r} \psi(m) \quad \text { if } r<0
$$

(b) for all $p>0$,

$$
\psi\left(|x|^{p}\right) \leq C(p) \psi(|x|) \leq C(p) \psi(1+|x|) .
$$

We will consider the following conditions.

$\left(\mathrm{H}_{1}\right) \sum_{n=1}^{\infty} q^{s / r}(n)<\infty$ for some $r \in\left(3 \cdot 2^{k-1}, 4 \cdot 2^{k-1}\right]$ and $r>\frac{\alpha p-1}{\alpha-1 / 2}$, where integer number $k \geq 1$ if $\alpha>1 / 2, \alpha p>1$ and $p \geq 2$.

$\left(\mathrm{H}_{2}\right) \sum_{n=1}^{\infty} q^{2}(n)<\infty$ if $\alpha>1 / 2, \alpha p>1$ and $1 \leq p<2$ or $\alpha>1 / 2$ and $\alpha p=1$.

Theorem 3.1 Let $\alpha>\frac{1}{2}$ and $\alpha p \geq 1$. Assume that $X_{n i}, i, n \in \mathbb{N}$, are an array of rowwise AANA random variables which are stochastically dominated by a random variable $X, a_{n i}$, $i, n \in \mathbb{N}$, are an array of real numbers with $\sum_{i=1}^{n}\left|a_{n i}\right|^{q}=O(n)$ for some $q>\max \left\{\frac{\alpha p-1}{\alpha-1 / 2}, 2\right\}$. Let $E X_{n i}=0$ for all $i, n \in \mathbb{N}$, if $p \geq 1$ and the conditions $\left(\mathrm{H}_{1}\right)$ and $\left(\mathrm{H}_{2}\right)$ are satisfied. If

$$
E|X|^{p} \psi(|X|)<\infty
$$

then

$$
\sum_{n=1}^{\infty} n^{\alpha p-2} \psi(n) P\left(\max _{1 \leq j \leq n}\left|\sum_{i=1}^{j} a_{n i} X_{n i}\right|>\varepsilon n^{\alpha}\right)<\infty \quad \text { for all } \varepsilon>0 .
$$

Proof Without loss of generality, we can assume that $a_{n i}>0$ for all $i, n \in \mathbb{N}$. For fixed $n \in \mathbb{N}$, let $X_{n i}^{\prime}=-n^{\alpha} I\left(X_{n i}<-n^{\alpha}\right)+X_{n i} I\left(\left|X_{n i}\right| \leq n^{\alpha}\right)+n^{\alpha} I\left(X_{n i}>n^{\alpha}\right)$ and $X_{n i}^{\prime \prime}=X_{n i}-X_{n i}^{\prime}, i \geq 1$. We will consider the following three cases.

(i) Let $p>1$. It is easy to check that

$$
\begin{aligned}
\sum_{n=1}^{\infty} & n^{\alpha p-2} \psi(n) P\left(\max _{1 \leq j \leq n}\left|\sum_{i=1}^{j} a_{n i} X_{n i}\right|>\varepsilon n^{\alpha}\right) \\
\leq & \sum_{n=1}^{\infty} n^{\alpha p-2} \psi(n) P\left(\max _{1 \leq j \leq n}\left|\sum_{i=1}^{j} a_{n i}\left(X_{n i}^{\prime}-E X_{n i}^{\prime}\right)\right|>\varepsilon n^{\alpha} / 2\right) \\
& +\sum_{n=1}^{\infty} n^{\alpha p-2} \psi(n) P\left(\max _{1 \leq j \leq n}\left|\sum_{i=1}^{j} a_{n i}\left(X_{n i}^{\prime \prime}-E X_{n i}^{\prime \prime}\right)\right|>\varepsilon n^{\alpha} / 2\right) \\
:= & I^{*}+J^{*} .
\end{aligned}
$$


By $C_{r}$ inequality and $\sum_{i=1}^{n} a_{n i}^{q}=O(n)$, it is easy to check that for all $0<\gamma \leq q$,

$$
\frac{1}{n} \sum_{i=1}^{n} a_{n i}^{\gamma} \leq\left(\frac{1}{n} \sum_{i=1}^{n} a_{n i}^{q}\right)^{\gamma / q}=O(1)
$$

For $J^{*}$, noting that $\left|X_{n i}^{\prime \prime}\right| \leq\left|X_{n i}\right| I\left(\left|X_{n i}\right|>n^{\alpha}\right)$, we have by Markov's inequality, Lemma 2.1 and (3.3), that

$$
\begin{aligned}
J^{*} & \leq C \sum_{n=1}^{\infty} n^{\alpha p-2-\alpha} \psi(n) \sum_{i=1}^{n} a_{n i} E\left|X_{n i}^{\prime \prime}\right| \\
& \leq C \sum_{n=1}^{\infty} n^{\alpha p-2-\alpha} \psi(n) \sum_{i=1}^{n} a_{n i} E\left|X_{n i}\right| I\left(\left|X_{n i}\right|>n^{\alpha}\right) \\
& \leq C \sum_{n=1}^{\infty} n^{\alpha p-1-\alpha} \psi(n) E|X| I\left(|X|>n^{\alpha}\right) \\
& =C \sum_{n=1}^{\infty} n^{\alpha p-1-\alpha} \psi(n) \sum_{j=n}^{\infty} E|X| I\left(j<|X|^{1 / \alpha} \leq j+1\right) \\
& =C \sum_{j=1}^{\infty} E|X| I\left(j<|X|^{1 / \alpha} \leq j+1\right) \sum_{n=1}^{j} n^{\alpha p-1-\alpha} \psi(n) \\
& \leq C \sum_{j=1}^{\infty} j^{\alpha p-\alpha} \psi(j) E|X| I\left(j<|X|^{1 / \alpha} \leq j+1\right) \\
& \leq C E|X|^{p} \psi\left(|X|^{1 / \alpha}\right) \leq C E|X|^{p} \psi(|X|)<\infty .
\end{aligned}
$$

For $I^{*}$, note that for every $n \in \mathbb{N}, a_{n i} X_{n i}^{\prime}-E a_{n i} X_{n i}^{\prime}, i \in \mathbb{N}$, are AANA random variables from Lemma 2.2. By Markov's inequality, Lemma 2.3 and Jensen's inequality, we have that for any $r \geq 2$,

$$
\begin{aligned}
I^{*} & \leq C_{r} \sum_{n=1}^{\infty} n^{\alpha p-2-\alpha r} \psi(n) E\left(\max _{1 \leq j \leq n}\left|\sum_{i=1}^{j}\left(a_{n i} X_{n i}^{\prime}-E a_{n i} X_{n i}^{\prime}\right)\right|^{r}\right) \\
& \leq C_{r} \sum_{n=1}^{\infty} n^{\alpha p-2-\alpha r} \psi(n) \sum_{i=1}^{n} a_{n i}^{r} E\left|X_{n i}^{\prime}\right|^{r}+C_{r} \sum_{n=1}^{\infty} n^{\alpha p-2-\alpha r} \psi(n)\left(\sum_{i=1}^{n} a_{n i}^{2} E\left(X_{n i}^{\prime}\right)^{2}\right)^{r / 2} \\
& :=I_{1}^{*}+I_{2}^{*} .
\end{aligned}
$$

We consider the following three cases.

Case $1 . \alpha>1 / 2, \alpha p>1$ and $p \geq 2$.

Take $r=q$. By $q>\max \left\{\frac{\alpha p-1}{\alpha-1 / 2}, 2\right\}$, it follows that $q>p$ and $\alpha p-2-\alpha q+q / 2<-1$.

For $I_{1}^{*}$, we have by $C_{r}$ inequality that

$$
\begin{aligned}
I_{1}^{*} & \leq C \sum_{n=1}^{\infty} n^{\alpha p-2-\alpha q} \psi(n) \sum_{i=1}^{n} a_{n i}^{q}\left(E\left|X_{n i}\right|^{q} I\left(\left|X_{n i}\right| \leq n^{\alpha}\right)+n^{\alpha q} P\left(\left|X_{n i}\right|>n^{\alpha}\right)\right) \\
& \leq C \sum_{n=1}^{\infty} n^{\alpha p-2-\alpha q} \psi(n) \sum_{i=1}^{n} a_{n i}^{q}\left(E|X|^{q} I\left(|X| \leq n^{\alpha}\right)+n^{\alpha q} P\left(|X|>n^{\alpha}\right)\right)
\end{aligned}
$$




$$
\begin{aligned}
& \leq C \sum_{n=1}^{\infty} n^{\alpha p-1-\alpha q} \psi(n) E|X|^{q} I\left(|X| \leq n^{\alpha}\right)+C \sum_{n=1}^{\infty} n^{\alpha p-1-\alpha} \psi(n) E|X| I\left(|X|>n^{\alpha}\right) \\
& \leq C \sum_{n=1}^{\infty} n^{\alpha(p-q)-1} \psi(n) \sum_{j=1}^{n} j^{\alpha q} P\left(j-1<|X|^{1 / \alpha} \leq j\right)+C E|X|^{p} \psi(|X|) \\
& \leq C \sum_{j=1}^{\infty} j^{\alpha q} P\left(j-1<|X|^{1 / \alpha} \leq j\right) \sum_{n=j}^{\infty} n^{\alpha(p-q)-1} \psi(n)+C E|X|^{p} \psi(|X|) \\
& \leq C \sum_{j=1}^{\infty} j^{\alpha p} \psi(j) P\left(j-1<|X|^{1 / \alpha} \leq j\right)+C E|X|^{p} \psi(|X|) \\
& \leq C E|X|^{p} \psi\left(|X|^{1 / \alpha}\right) \leq C E|X|^{p} \psi(|X|)<\infty .
\end{aligned}
$$

For $I_{2}^{*}$, note that $E X^{2}<\infty$ if $E|X|^{p} \psi(|X|)<\infty$ for $p \geq 2$. We have by (3.6) that

$$
\begin{aligned}
I_{2}^{*} & \leq C \sum_{n=1}^{\infty} n^{\alpha p-2-\alpha q} \psi(n)\left(\sum_{i=1}^{n} a_{n i}^{2} E X_{n i}^{2}\right)^{q / 2} \\
& \leq C \sum_{n=1}^{\infty} n^{\alpha p-2-\alpha q} \psi(n)\left(\sum_{i=1}^{n} a_{n i}^{2} E X^{2}\right)^{q / 2} \\
& \leq C \sum_{n=1}^{\infty} n^{\alpha p-2-\alpha q+q / 2} \psi(n)<\infty .
\end{aligned}
$$

Case 2. $\alpha>1 / 2, \alpha p>1$ and $1<p<2$.

Take $r=2$. Similar to the proofs of (3.8), (3.9) and (3.7), we have that

$$
\begin{aligned}
I^{*} & \leq C \sum_{n=1}^{\infty} n^{\alpha p-2-2 \alpha} \psi(n) \sum_{i=1}^{n} a_{n i}^{2}\left(E X_{n i}^{2} I\left(\left|X_{n i}\right| \leq n^{\alpha}\right)+n^{2 \alpha} P\left(\left|X_{n i}\right|>n^{\alpha}\right)\right) \\
& \leq C \sum_{n=1}^{\infty} n^{\alpha p-1-2 \alpha} \psi(n) E X^{2} I\left(|X| \leq n^{\alpha}\right)+C \sum_{n=1}^{\infty} n^{\alpha p-1-\alpha} \psi(n) E|X| I\left(|X|>n^{\alpha}\right) \\
& <\infty .
\end{aligned}
$$

Case 3. $\alpha>1 / 2, \alpha p=1$ and $p>1$.

Take $r=2$. Note that $1 / 2<\alpha<1$ if $\alpha p=1$. Similar to the proof of (3.10), it follows that $I^{*}<\infty$.

(ii) Let $p=1$. Note that $\alpha \geq 1$ from $\alpha p \geq 1$. By $E X_{n i}=0$ for $i, n \in \mathbb{N}$, Lemma 2.1, (3.6) and (3.4), we have that

$$
\begin{aligned}
n^{-\alpha} \max _{1 \leq j \leq n}\left|\sum_{i=1}^{j} a_{n i} E X_{n i}^{\prime}\right| & \leq n^{-\alpha} \sum_{i=1}^{n} a_{n i} E\left|X_{n i}\right| I\left(\left|X_{n i}\right|>n^{\alpha}\right) \\
& \leq n^{1-\alpha} E|X| I\left(|X|>n^{\alpha}\right) \rightarrow 0 \text { as } n \rightarrow \infty
\end{aligned}
$$

Hence for $n$ large enough, we have

$$
n^{-\alpha} \max _{1 \leq j \leq n}\left|\sum_{i=1}^{j} a_{n i} X_{n i}^{\prime}\right|<\frac{\varepsilon}{2} .
$$


It follows that

$$
\begin{aligned}
\sum_{n=1}^{\infty} n^{\alpha-2} \psi(n) P\left(\max _{1 \leq j \leq n}\left|\sum_{i=1}^{j} a_{n i} X_{n i}\right|>\varepsilon n^{\alpha}\right) \\
\leq \sum_{n=1}^{\infty} n^{\alpha-2} \psi(n) \sum_{i=1}^{n} P\left(\left|X_{n i}\right|>n^{\alpha}\right) \\
\quad+\sum_{n=1}^{\infty} n^{\alpha-2} \psi(n) P\left(\max _{1 \leq j \leq n}\left|\sum_{i=1}^{j} a_{n i} X_{n i}^{\prime}\right|>\varepsilon n^{\alpha}\right) \\
\leq C \sum_{n=1}^{\infty} n^{\alpha-1} \psi(n) P\left(|X|>n^{\alpha}\right) \\
\quad+C \sum_{n=1}^{\infty} n^{\alpha-2} \psi(n) P\left(\max _{1 \leq j \leq n}\left|\sum_{i=1}^{j} a_{n i}\left(X_{n i}^{\prime}-E X_{n i}^{\prime}\right)\right|>\frac{\varepsilon n^{\alpha}}{2}\right) \\
:=C I_{1}+C I_{2} .
\end{aligned}
$$

For $I_{1}$, we have by (3.1) and (3.4) that

$$
\begin{aligned}
I_{1} & =\sum_{n=1}^{\infty} n^{\alpha-1} \psi(n) \sum_{i=n}^{\infty} P\left(i^{\alpha}<|X| \leq(i+1)^{\alpha}\right) \\
& =\sum_{i=1}^{\infty} P\left(i^{\alpha}<|X| \leq(i+1)^{\alpha}\right) \sum_{n=1}^{i} n^{\alpha-1} \psi(n) \\
& \leq C \sum_{i=1}^{\infty} P\left(i^{\alpha}<|X| \leq(i+1)^{\alpha}\right) i^{\alpha} \psi(i) \\
& \leq C E|X| \psi\left(|X|^{1 / \alpha}\right) \leq C E|X| \psi(|X|)<\infty .
\end{aligned}
$$

For $I_{2}$, we have by Markov's inequality, Lemma 2.3, Lemma 2.1, (3.2) and (3.3) that

$$
\begin{aligned}
I_{2} & \leq C \sum_{n=1}^{\infty} n^{-\alpha-2} \psi(n) E \max _{1 \leq j \leq n}\left(\sum_{i=1}^{j} a_{n i}\left(X_{n i}^{\prime}-E X_{n i}^{\prime}\right)\right)^{2} \\
& \leq C \sum_{n=1}^{\infty} n^{-\alpha-2} \psi(n) \sum_{i=1}^{n} a_{n i}^{2} E\left(X_{n i}^{\prime}\right)^{2} \\
& =C \sum_{n=1}^{\infty} n^{-\alpha-2} \psi(n)\left\{\sum_{i=1}^{n} a_{n i}^{2} E X_{n i}^{2} I\left(\left|X_{n i}\right| \leq n^{\alpha}\right)+n^{2 \alpha} \sum_{i=1}^{n} a_{n i}^{2} P\left(\left|X_{n i}\right|>n^{\alpha}\right)\right\} \\
& \leq C \sum_{n=1}^{\infty} n^{-\alpha-1} \psi(n) E X^{2} I\left(|X| \leq n^{\alpha}\right)+C \sum_{n=1}^{\infty} n^{\alpha-1} \psi(n) P\left(|X|>n^{\alpha}\right) \\
& =C \sum_{n=1}^{\infty} n^{-\alpha-1} \psi(n) \sum_{k=1}^{n} E X^{2} I\left((k-1)^{\alpha}<|X| \leq k^{\alpha}\right)+C \\
& =C \sum_{k=1}^{\infty} E X^{2} I\left((k-1)^{\alpha}<|X| \leq k^{\alpha}\right) \sum_{n=k}^{\infty} n^{-\alpha-1} \psi(n)+C
\end{aligned}
$$




$$
\begin{aligned}
& \leq C \sum_{k=1}^{\infty} k^{-\alpha} \psi(k) E X^{2} I\left((k-1)^{\alpha}<|X| \leq k^{\alpha}\right)+C \\
& \leq C E|X| \psi(|X|)+C<\infty
\end{aligned}
$$

By (3.12)-(3.14), (3.5) holds for the case $p=1$.

(iii) Let $0<p<1$. Denote

$$
\sum_{i=1}^{j} a_{n i} X_{n i}=\sum_{i=1}^{j} a_{n i} X_{n i} I\left(\left|X_{n i}\right| \leq n^{\alpha}\right)+\sum_{i=1}^{j} a_{n i} X_{n i} I\left(\left|X_{n i}\right|>n^{\alpha}\right)=: S_{n j}^{\prime}+S_{n j}^{\prime \prime} .
$$

Noting that $E|X|^{p} \psi(|X|)<\infty$, we have by Markov's inequality, Lemma 2.1 and (3.2)-(3.6), that

$$
\begin{aligned}
\sum_{n=1}^{\infty} n^{\alpha p-2} \psi(n) P\left(\max _{1 \leq j \leq n}\left|S_{n j}^{\prime}\right|>\varepsilon n^{\alpha}\right) \\
\leq \varepsilon^{-1} \sum_{n=1}^{\infty} n^{\alpha p-2-\alpha} \psi(n) E\left(\max _{1 \leq j \leq n}\left|\sum_{i=1}^{j} a_{n i} X_{n i} I\left(\left|X_{n i}\right| \leq n^{\alpha}\right)\right|\right) \\
\leq \varepsilon^{-1} \sum_{n=1}^{\infty} n^{\alpha p-2-\alpha} \psi(n) \sum_{i=1}^{n} a_{n i} E\left|X_{n i}\right| I\left(\left|X_{n i}\right| \leq n^{\alpha}\right) \\
\leq C \varepsilon^{-1} \sum_{n=1}^{\infty} n^{\alpha p-1-\alpha} \psi(n) E|X| I\left(|X| \leq n^{\alpha}\right)+C \varepsilon^{-1} \sum_{n=1}^{\infty} n^{\alpha p-1} \psi(n) P\left(|X|>n^{\alpha}\right) \\
=C \varepsilon^{-1} \sum_{n=1}^{\infty} n^{\alpha p-1-\alpha} \psi(n) \sum_{j=1}^{n} E|X| I\left(j-1<|X|^{1 / \alpha} \leq j\right) \\
\quad+C \varepsilon^{-1} \sum_{n=1}^{\infty} n^{\alpha p-1} \psi(n) \sum_{j=n}^{\infty} P\left(j<|X|^{1 / \alpha} \leq j+1\right) \\
\leq C \varepsilon^{-1} \sum_{j=1}^{\infty} j^{\alpha} P\left(j-1<|X|^{1 / \alpha} \leq j\right) \sum_{n=j}^{\infty} n^{\alpha p-1-\alpha} \psi(n) \\
\quad+C \varepsilon^{-1} \sum_{j=1}^{\infty} P\left(j<|X|^{1 / \alpha} \leq j+1\right) \sum_{n=1}^{j} n^{\alpha p-1} \psi(n) \\
\leq C \varepsilon^{-1} \sum_{j=1}^{\infty} j^{\alpha p} \psi(j) P\left(j-1<|X|^{1 / \alpha} \leq j\right)+C \varepsilon^{-1} \sum_{j=1}^{\infty} j^{\alpha p} \psi(j) P\left(j<|X|^{1 / \alpha} \leq j+1\right) \\
\leq C E|X|^{p} \psi\left(|X|^{1 / \alpha}\right) \leq C E|X|^{p} \psi(|X|)<\infty
\end{aligned}
$$

and

$$
\begin{aligned}
& \sum_{n=1}^{\infty} n^{\alpha p-2} \psi(n) P\left(\max _{1 \leq j \leq n}\left|S_{n j}^{\prime \prime}\right|>\varepsilon n^{\alpha}\right) \\
& \quad \leq \varepsilon^{-p / 2} \sum_{n=1}^{\infty} n^{\alpha p / 2-2} \psi(n) E\left(\max _{1 \leq j \leq n}\left|\sum_{i=1}^{j} a_{n i} X_{n i} I\left(\left|X_{n i}\right|>n^{\alpha}\right)\right|\right)^{p / 2}
\end{aligned}
$$




$$
\begin{aligned}
& \leq \varepsilon^{-p / 2} \sum_{n=1}^{\infty} n^{\alpha p / 2-2} \psi(n) \sum_{i=1}^{n} a_{n i}^{p / 2} E\left|X_{n i}\right|^{p / 2} I\left(\left|X_{n i}\right|>n^{\alpha}\right) \\
& \leq C \varepsilon^{-p / 2} \sum_{n=1}^{\infty} n^{\alpha p / 2-1} \psi(n) E|X|^{p / 2} I\left(|X|>n^{\alpha}\right) \\
& =C \varepsilon^{-p / 2} \sum_{n=1}^{\infty} n^{\alpha p / 2-1} \psi(n) \sum_{j=n}^{\infty} E|X|^{p / 2} I\left(j<|X|^{1 / \alpha} \leq j+1\right) \\
& \leq C \varepsilon^{-p / 2} \sum_{j=1}^{\infty} j^{\alpha p / 2} P\left(j<|X|^{1 / \alpha} \leq j+1\right) \sum_{n=1}^{j} n^{\alpha p / 2-1} \psi(n) \\
& \leq C \varepsilon^{-p / 2} \sum_{j=1}^{\infty} j^{\alpha p} \psi(j) P\left(j-1<|X|^{1 / \alpha} \leq j\right) \\
& \leq C E|X|^{p} \psi\left(|X|^{1 / \alpha}\right) \leq C E|X|^{p} \psi(|X|)<\infty .
\end{aligned}
$$

Hence (3.15)-(3.17) imply (3.5). From all the statements above, we have proved (3.5).

Remark 3.1 Taking $\psi(x) \equiv 1$ and $a_{n i} \equiv 1$ in Theorem 3.1, we can get (i) of Theorem B; meanwhile, relax the mixing coefficient condition $\sum_{n=1}^{\infty} q^{s / r}(n)<\infty$ to $\sum_{n=1}^{\infty} q^{2}(n)<\infty$ for the case $\alpha>1 / 2, \alpha p>1$ and $1<p<2$. In addition, we extend the case $1 / 2<\alpha \leq 1, p>1$ and $\alpha p>1$ to the case $\alpha>1 / 2, \alpha p \geq 1$. Taking $\psi(x) \equiv 1, a_{n i} \equiv 1$ and $\alpha=1, p=1$ in Theorem 3.1, we can get (ii) of Theorem $B$ and weaken the condition $E|X| \log |X|<\infty$ to the condition $E|X|<\infty$. Hence we extend and improve the corresponding results of [10].

Remark 3.2 Under the conditions of Theorem 3.1, we have that for $p>1$,

$$
\sum_{n=1}^{\infty} n^{\alpha p-2-\alpha} \psi(n) E\left(\max _{1 \leq j \leq n}\left|\sum_{i=1}^{j} a_{n i} X_{n i}\right|-\varepsilon n^{\alpha}\right)^{+}<\infty .
$$

In fact, by Lemma 2.4 with $r \geq 2$, we get

$$
\begin{aligned}
\sum_{n=1}^{\infty} n^{\alpha p-2-\alpha} \psi(n) E\left(\max _{1 \leq j \leq n}\left|\sum_{i=1}^{j} a_{n i} X_{n i}\right|-\varepsilon n^{\alpha}\right)^{+} \\
\leq C \sum_{n=1}^{\infty} n^{\alpha p-2-\alpha r} \psi(n) E\left(\max _{1 \leq j \leq n}\left|\sum_{i=1}^{j}\left(a_{n i} X_{n i}^{\prime}-E a_{n i} X_{n i}^{\prime}\right)\right|\right)^{r} \\
\quad+\sum_{n=1}^{\infty} n^{\alpha p-2-\alpha} \psi(n) E\left(\max _{1 \leq j \leq n}\left|\sum_{i=1}^{j}\left(a_{n i} X_{n i}^{\prime \prime}-E a_{n i} X_{n i}^{\prime \prime}\right)\right|\right) .
\end{aligned}
$$

By the process of the proof of Theorem 3.1 in the case $p>1$, it follows that (3.18) holds.

Similar to the proof of Theorem 3.1, we can get easily the following result.

Theorem 3.2 Let $\alpha>\frac{1}{2}$ and $\alpha p \geq 1$. Let $X_{n}, n \in \mathbb{N}$, be AANA random variables which are stochastically dominated by a random variable $X$. Assume that $a_{n}, n \in \mathbb{N}$, are real numbers 
with $\sum_{i=1}^{n}\left|a_{i}\right|^{q}=O(n)$ for some $q>\max \left\{\frac{\alpha p-1}{\alpha-1 / 2}, 2\right\}$, the conditions $\left(\mathrm{H}_{1}\right)$ and $\left(\mathrm{H}_{2}\right)$ are satisfied. If (3.4) holds, then

$$
\sum_{n=1}^{\infty} n^{\alpha p-2} \psi(n) P\left(\max _{1 \leq j \leq n}\left|\sum_{i=1}^{j} a_{i} X_{i}\right|>\varepsilon n^{\alpha}\right)<\infty \quad \text { for all } \varepsilon>0 .
$$

Remark 3.3 Similar to Remark 3.1, taking $\psi(x) \equiv 1$ and $a_{i} \equiv 1$ in Theorem 3.2, we can get (i) of Theorem 3.4 in [10]; meanwhile, relax the mixing coefficient condition $\sum_{n=1}^{\infty} q^{s / r}(n)<$ $\infty$ to $\sum_{n=1}^{\infty} q^{2}(n)<\infty$ for the case $\alpha>1 / 2, \alpha p>1$ and $1<p<2$. In addition, we extend the case $1 / 2<\alpha \leq 1, p>1$ and $\alpha p>1$ to the case $\alpha>1 / 2, \alpha p \geq 1$. Taking $\psi(x) \equiv 1, a_{n i} \equiv 1$ and $\alpha=1, p=1$ in Theorem 3.2, we can get (ii) of Theorem 3.4 in [10] and weaken the condition $E|X| \log |X|<\infty$ to the condition $E|X|<\infty$. Hence, we extend and improve the corresponding results of [10].

In the following, we give the Marcinkiewicz-Zygmund type strong law of large numbers of weights sums on AANA random variables.

Corollary 3.1 Let $\alpha>\frac{1}{2}$ and $\alpha p \geq 1$. Let $X_{n}, n \in \mathbb{N}$, be AANA random variables which are stochastically dominated by a random variable $X$. Assume that $a_{n}, n \in \mathbb{N}$ are real numbers with $\sum_{i=1}^{n}\left|a_{i}\right|^{q}=O(n)$ for some $q>\max \left\{\frac{\alpha p-1}{\alpha-1 / 2}, 2\right\}$, the conditions $\left(\mathrm{H}_{1}\right)$ and $\left(\mathrm{H}_{2}\right)$ are satisfied. If $E|X|^{p}<\infty$, then

$$
\sum_{n=1}^{\infty} n^{\alpha p-2} P\left(\max _{1 \leq j \leq n}\left|\sum_{i=1}^{j} a_{i} X_{i}\right|>\varepsilon n^{\alpha}\right)<\infty
$$

and

$$
n^{-\alpha} \sum_{i=1}^{n} a_{i} X_{i} \rightarrow 0 \quad \text { a.s. } n \rightarrow \infty .
$$

Further, for $p>1$,

$$
\sum_{n=1}^{\infty} n^{\alpha p-2-\alpha} E\left(\max _{1 \leq j \leq n}\left|\sum_{i=1}^{j} a_{i} X_{i}\right|-\varepsilon n^{\alpha}\right)^{+}<\infty .
$$

Proof Taking $\psi(x)=1$ in Theorem 3.2, we get (3.19) easily. Similar to the proof of (3.18), (3.21) is obtained immediately. We only need to prove (3.20).

By (3.20), it follows that for all $\varepsilon>0$,

$$
\begin{aligned}
\infty & >\sum_{n=1}^{\infty} n^{\alpha p-2} P\left(\max _{1 \leq j \leq n}\left|\sum_{i=1}^{j} a_{i} X_{i}\right|>\varepsilon n^{\alpha}\right)=\sum_{k=0}^{\infty} \sum_{n=2^{k}}^{2^{k+1}-1} n^{\alpha p-2} P\left(\max _{1 \leq j \leq n}\left|\sum_{i=1}^{j} a_{i} X_{i}\right|>\varepsilon n^{\alpha}\right) \\
& \geq \begin{cases}\sum_{k=0}^{\infty}\left(2^{k}\right)^{\alpha p-2} 2^{k} P\left(\max _{1 \leq j \leq 2^{k}}\left|\sum_{i=1}^{j} a_{i} X_{i}\right|>\varepsilon 2^{(k+1) \alpha}\right) & \text { if } \alpha p \geq 2, \\
\sum_{k=0}^{\infty}\left(2^{k+1}\right)^{\alpha p-2} 2^{k} P\left(\max _{1 \leq j \leq 2^{k}}\left|\sum_{i=1}^{j} a_{i} X_{i}\right|>\varepsilon 2^{(k+1) \alpha}\right) & \text { if } 1 \leq \alpha p<2\end{cases} \\
& \geq \begin{cases}\sum_{k=0}^{\infty} P\left(\max _{1 \leq j \leq 2^{k}}\left|\sum_{i=1}^{j} a_{i} X_{i}\right|>\varepsilon 2^{(k+1) \alpha}\right) & \text { if } \alpha p \geq 2, \\
\frac{1}{2} \sum_{k=0}^{\infty} P\left(\max _{1 \leq j \leq 2^{k}}\left|\sum_{i=1}^{j} a_{i} X_{i}\right|>\varepsilon 2^{(k+1) \alpha}\right) & \text { if } 1 \leq \alpha p<2 .\end{cases}
\end{aligned}
$$


By the Borel-Cantelli lemma, we obtain that

$$
\frac{\max _{1 \leq j \leq 2^{k}}\left|\sum_{i=1}^{j} a_{i} X_{i}\right|}{2^{(k+1) \alpha}} \rightarrow 0 \quad \text { a.s. } k \rightarrow \infty
$$

For all positive integers $n$, there exists a positive integer $k$ such that $2^{k-1} \leq n \leq 2^{k}$. We have by (3.22) that

$$
n^{-\alpha}\left|\sum_{i=1}^{n} a_{i} X_{i}\right| \leq \max _{2^{k-1} \leq n \leq 2^{k}} n^{-\alpha}\left|\sum_{i=1}^{n} a_{i} X_{i}\right| \leq \frac{2^{\alpha} \max _{1 \leq j \leq 2^{k}}\left|\sum_{i=1}^{j} a_{i} X_{i}\right|}{2^{(k+1) \alpha}} \rightarrow 0 \quad \text { a.s. } k \rightarrow \infty
$$

which implies that

$$
n^{-\alpha} \sum_{i=1}^{n} a_{i} X_{i} \rightarrow 0 \quad \text { a.s. } n \rightarrow \infty
$$

This completes the proof of the corollary.

Remark 3.4 Taking $a_{n} \equiv 1$ in Corollary 3.1, we can get the Baum-Katz result on AANA random variables. Comparing with Theorem 3.4 and Corollary 3.5 of [10], Corollary 3.1 relaxes the mixing coefficient condition $\sum_{n=1}^{\infty} q^{s / r}(n)<\infty$ to $\sum_{n=1}^{\infty} q^{2}(n)<\infty$ for the case $\alpha>1 / 2, \alpha p>1$ and $1<p<2$. In addition, we also consider the case $\alpha p=1$ and the case $\alpha p \geq$ 1 and $0<p \leq 1$. Taking $\alpha=1$ and $p=2$ in Corollary 3.1, we can get the Hsu-Robbins-type theorem (see [13]) on AANA random variables. Taking $\alpha=1$ and $p=1$ in Corollary 3.1, we improve (ii) of Theorem 3.4 and (ii) of Corollary 3.5 in [10].

\section{Competing interests \\ The authors declare that they have no competing interests.}

\section{Authors' contributions}

All authors read and approved the final manuscript.

\section{Acknowledgements}

The authors are most grateful to the editor Andrei Volodin and anonymous referees for careful reading of the manuscript and valuable suggestions which helped in significantly improving an earlier version of this paper. The work was supported by the National Natural Science Foundation of China (11171001, 11201001), Doctoral Research Start-up Funds Projects of Anhui University and Natural Science Foundation of Anhui Province (1308085QA03, 1208085QA03).

Received: 7 March 2013 Accepted: 18 July 2013 Published: 2 August 2013

\section{References}

1. Block, HW, Savits, TH, Shaked, M: Some concepts of negative dependence. Ann. Probab. 10(3), 765-772 (1982)

2. Joag-Dev, K, Proschan, F: Negative association of random variables, with applications. Ann. Stat. 11(1), $286-295$ (1983)

3. Chandra, TK, Ghosal, S: Extensions of the strong law of large numbers of Marcinkiewicz and Zygmund for dependent variables. Acta Math. Hung. 71(4), 327-336 (1996)

4. Chandra, TK, Ghosal, S: The strong law of large numbers for weighted averages under dependence assumptions. J. Theor. Probab. 9(3), 797-809 (1996)

5. Wang, Y, Yan, J, Cheng, F, Su, C: The strong law of large numbers and the law of iterated logarithm for product sums of NA and AANA random variables. Southeast Asian Bull. Math. 27(2), 369-384 (2003)

6. Ko, M-H, Kim, T-S, Lin, Z: The Hàjeck-Rènyi inequality for the AANA random variables and its applications. Taiwan. J. Math. 9(1), 111-122 (2005)

7. Yuan, DM, An, J: Rosenthal type inequalities for asymptotically almost negatively associated random variables and applications. Sci. China Ser. A 52(9), 1887-1904 (2009)

8. Wang, XJ, Hu, SH, Yang, WZ: Convergence properties for asymptotically almost negatively associated sequence. Discrete Dyn. Nat. Soc. 2010, Article ID 218380 (2010)

9. Wang, XJ, Hu, SH, Yang, WZ: Complete convergence for arrays of rowwise asymptotically almost negatively associated random variables. Discrete Dyn. Nat. Soc. 2011, Article ID 717126 (2011) 
10. Wang, XJ, Hu, SH, Yang, WZ, Wang, XH: On complete convergence of weighted sums for arrays of rowwise asymptotically almost negatively associated random variables. Abstr. Appl. Anal. 2012, Article ID 315138 (2012)

11. Yang, WZ, Wang, XJ, Ling, NX, Hu, SH: On complete convergence of moving average process for AANA sequence. Discrete Dyn. Nat. Soc. 2012, Article ID 863931 (2012)

12. Shen, AT, Wu, RC: Strong and weak convergence for asymptotically almost negatively associated random variables. Discrete Dyn. Nat. Soc. 2013, Article ID 235012 (2013)

13. Hsu, PL, Robbins, H: Complete convergence and the law of large numbers. Proc. Natl. Acad. Sci. USA 33(2), 25-31 (1947)

14. Erdös, P: On a theorem of Hsu and Robbins. Ann. Math. Stat. 20(2), 286-291 (1949)

15. Baum, LE, Katz, M: Convergence rates in the law of large numbers. Trans. Am. Math. Soc. 120(1), 108-123 (1965)

16. Peligrad, M: Convergence rates of the strong law for stationary mixing sequences. Z. Wahrscheinlichkeitstheor. Verw. Geb. 70(2), 307-314 (1985)

17. Shao, QM: A moment inequality and its applications. Acta Math. Sin. 31(6), 736-747 (1988)

18. Peligrad, M, Gut, A: Almost-sure results for a class of dependent random variables. J. Theor. Probab. 12(1), 87-104 (1999)

19. Kruglov, VM, Volodin, A, Hu, T-C: On complete convergence for arrays. Stat. Probab. Lett. 76, 1631-1640 (2006)

20. Wang, XJ, Hu, SH: Some Baum-Katz type results for $\varphi$-mixing random variables with different distributions. Rev. R. Acad. Cienc. Exactas Fís. Nat., Ser. a Mat. (Ed. Impr.) 106(2), 321-331 (2012)

21. Shen, AT, Wang, $\mathrm{XH}$, Ling, JM: On complete convergence for non-stationary $\varphi$-mixing random variables. Commun. Stat., Theory Methods (2012). doi:10.1080/03610926.2012.725501

22. Wang, XJ, Hu, SH, Yang, WZ, Wang, XH: Convergence rates in the strong law of large numbers for martingale difference sequences. Abstr. Appl. Anal. 2012, Article ID 572493 (2012)

23. Hu, T-C, Ordóñez Cabrera, M, Sung, SH, Volodin, A: Complete convergence for arrays of rowwise independent random variables. Commun. Korean Math. Soc. 18, 375-383 (2003)

24. Sung, SH, Volodin, A, Hu, T-C: More on complete convergence for arrays. Stat. Probab. Lett. 71, $303-311$ (2005)

25. Kuczmaszewska, A: On complete convergence for arrays of rowwise dependent random variables. Stat. Probab. Lett. 77, 1050-1060 (2007)

26. Kuczmaszewska, A: On complete convergence for arrays of rowwise negatively associated random variables. Stat. Probab. Lett. 79, 116-124 (2009)

27. Chen, P, Hu, T-C, Liu, X, Volodin, A: On complete convergence for arrays of row-wise negatively associated random variables. Theory Probab. Appl. 52, 323-328 (2008)

28. Kruglov, VM: Complete convergence for maximal sums of negatively associated random variables. J. Probab. Stat. 2010, Article ID 764043 (2010)

29. Zhou, XC, Lin, JG: On complete convergence for arrays of rowwise $\rho$-mixing random variables and its applications. J. Inequal. Appl. 2010, Article ID 769201 (2010)

30. Guo, ML: Complete moment convergence of weighted sums for arrays of rowwise $\varphi$-mixing random variables. Int. J. Math. Math. Sci. 2012, Article ID 730962 (2012)

31. Wu, QY: A complete convergence theorem for weighted sums of arrays of rowwise negatively dependent random variables. J. Inequal. Appl. 2012, Article ID 50 (2012)

32. Wu, QY: Probability Limit Theory for Mixed Sequence. China Sci. \& Technol. Press, Beijing (2006)

33. Sung, SH: Moment inequalities and complete moment convergence. J. Inequal. Appl. 2009, Article ID 271265 (2009)

34. Chen, PY, Volodin, A: Limiting behaviour of moving average processes under negative association assumption. Theory Probab. Math. Stat. 77, 165-176 (2008)

doi:10.1186/1029-242X-2013-359

Cite this article as: Wang et al.: A note on complete convergence of weighted sums for array of rowwise AANA random variables. Journal of Inequalities and Applications 2013 2013:359.

\section{Submit your manuscript to a SpringerOpen ${ }^{\circ}$ journal and benefit from:}

- Convenient online submission

Rigorous peer review

- Immediate publication on acceptance

- Open access: articles freely available online

- High visibility within the field

- Retaining the copyright to your article 